\title{
Review of the top 5 cardiology studies of 2011-12
}

\author{
Arden R. Barry, BSc, BSc(Pharm), ACPR, PharmD; Rosaleen Boswell, BScPharm, ACPR; \\ Ernest H. Law, BScPharm, ACPR; Dylan M. Pollmann, BScPharm; Mohamed A. Omar, \\ BScPharm, PhD; Margaret L. Ackman, BSc(Pharm), PharmD, ACPR, FCSHP
}

\section{Introduction}

Each year, hundreds of trials are published in various specialties, making it difficult for pharmacists in general practice to ensure they are reviewing the studies that are most likely to affect patient care. In this article, we present the 5 recent cardiovascular trials judged to have the greatest importance for pharmacists.

\section{Methods}

Through discussion with clinical pharmacist specialists, a list of cardiology trials with potential to influence practice, published in 2011 and 2012, was developed. We asked all Edmontonarea Alberta Health Services pharmacists who regularly practise in inpatient and/or ambulatory cardiology, as well as inpatient internal and family medicine pharmacists from local teaching hospitals, to rank the importance of these 18 trials using an online survey. Twenty pharmacists responded and the top 5 ranked trials are reviewed briefly below, including a statement regarding potential implications for practice.

Three of the 5 trials focus on atrial fibrillation (AF), as this disease affects approximately 350,000 Canadians and that number is expected to grow as the population ages. ${ }^{1}$ Two direct factor Xa inhibitors, rivaroxaban ${ }^{2}$ and apixaban, ${ }^{3}$ are compared with warfarin in individual trials. Due to more predictable anticoagulation, these agents offer the convenience of less laboratory monitoring (including no need for international normalized ratio [INR] monitoring) and fewer drug and food interactions in comparison to warfarin. However, the cost of these agents is substantially higher, there is an inability to reverse their anticoagulant effects and their short half-life makes adherence more crucial. It is important to note that patients with mechanical heart valves were excluded from these trials. We have also included a validation trial examining the predictive value of scoring systems to identify patients with nonvalvular AF who would benefit from anticoagulant therapy. ${ }^{4}$ Rivaroxaban has also been studied in patients with acute coronary syndromes to determine if it provides benefit when added to current standard therapy. ${ }^{5}$ The last trial examines the use of eplerenone in ambulatory heart failure (HF) patients with mild symptoms. ${ }^{6}$

For both acute coronary syndromes and AF, the benefit of preventing thrombotic events and death must be balanced against the risk of bleeding. Although there is variation in the bleeding definitions used in these trials, major bleeding is commonly defined as fatal bleeding, bleeding at critical sites such as the central nervous system, decreases in hemoglobin of $\geq 20 \mathrm{~g} / \mathrm{L}$ or the need for transfusion. Minor bleeding is usually considered bleeding that does not require invasive management, including transfusions. None of these trials evaluated "nuisance" bleeding, which, although not medically crucial, has the potential to affect adherence and patient quality of life. 


\section{ATRIAL FIBRILLATION}

ROCKET-AF: Rivaroxaban versus warfarin in nonvalvular atrial fibrillation ( $N$ Engl J Med 2011)

Background: The objective of this randomized, multicentre, double-blind, double-dummy, noninferiority trial was to investigate whether rivaroxaban was noninferior to warfarin for the prevention of stroke or systemic embolism in patients with nonvalvular $\mathrm{AF}^{2}$

Patients: Enrolled were patients with nonvalvular $\mathrm{AF}$ and a previous stroke, transient ischemic attack (TIA) or systemic embolism, or at high risk for stroke, as defined by a $\mathrm{CHADS}_{2}$ (congestive heart failure, hypertension, age $\geq 75$ years, diabetes mellitus, stroke) score of $\geq 2$. The exclusion criteria included patients at a high risk of bleeding or a creatinine clearance $(\mathrm{CrCl})<30 \mathrm{~mL} / \mathrm{min}$.

Intervention and control: Patients were randomized to either rivaroxaban $20 \mathrm{mg}$ once daily (15 mg daily for patients with a $\mathrm{CrCl}$ of $30-49 \mathrm{~mL} /$ $\mathrm{min}$ ) or dose-adjusted warfarin with a target INR of $2-3$.

Outcomes: The primary efficacy outcome was a composite of stroke (ischemic or hemorrhagic) and systemic embolism. The primary safety outcome was a composite of major and nonmajor clinically relevant bleeding.

Results: A total of 14,264 patients were enrolled and followed for a median of 1.9 years. Forty percent were female, with a median age of 73 years and a mean $\mathrm{CHADS}_{2}$ score of 3.5. For the warfarin group, the mean INR time in therapeutic range (TTR) was 55\%. The proportion of patients in whom the primary efficacy outcome occurred was $1.7 \%$ per year in the rivaroxaban group compared with $2.2 \%$ per year in the warfarin group by the per protocol analysis, which satisfied the criteria for noninferiority $(p<0.001)$. However, the study failed to demonstrate that rivaroxaban was superior to warfarin $(p=0.12)$. The primary safety outcome was not significantly different between groups (approximately 15 events per 100 patientyears; $p=0.44)$. Although rare, rivaroxaban had a significantly lower rate of intracranial hemorrhage (ICH) and fatal bleeding compared with warfarin; however, rivaroxaban had a 1.5 -fold higher rate of major gastrointestinal (GI) bleeding.
Implications for practice: This study supports rivaroxaban as a potential alternative to warfarin in patients with nonvalvular AF, as it was not inferior at reducing the incidence of stroke and systemic embolism with a similar risk of bleeding and a lower rate of $\mathrm{ICH}$. However, the results of this trial are limited by the $55 \%$ TTR in the warfarin group, which was much lower than in other trials ${ }^{3,7,8}$ and therefore may not have represented a fair comparison.

ARISTOTLE: Apixaban versus warfarin in patients with atrial fibrillation ( $N$ Engl J Med 2011)

Background: The purpose of this multicentre, randomized, double-blind, double-dummy, noninferiority trial was to determine whether apixaban was noninferior to warfarin at preventing stroke or systemic embolism in patients with nonvalvular $\mathrm{AF}^{3}$

Patients: Included were patients with nonvalvular $\mathrm{AF} /$ atrial flutter (AFL) and at least 1 risk factor for stroke, including age $\geq 75$ years, previous stroke or TIA, systemic embolism, symptomatic HF or left ventricular ejection fraction (LVEF) $\leq 40 \%$, diabetes mellitus or hypertension. Excluded were patients with severe renal dysfunction (serum creatinine $>221 \mathrm{mmol} / \mathrm{L}$ or $\mathrm{CrCl}<25 \mathrm{~mL} / \mathrm{min}$ ), a recent stroke or those receiving anticoagulation or dual antiplatelet therapy for another indication.

Intervention and control: The intervention group received apixaban $5 \mathrm{mg}$ twice daily, which was reduced to $2.5 \mathrm{mg}$ twice daily in patients $>80$ years of age, weight $\geq 60 \mathrm{~kg}$ or serum creatinine $\geq 133$ $\mathrm{mmol} / \mathrm{L}$. The control group received dose-adjusted warfarin with a target INR of 2-3.

Outcomes: The primary efficacy outcome was a composite of stroke (ischemic and hemorrhagic) and systemic embolism. The secondary efficacy outcome was death from any cause. The primary safety outcome was major bleeding.

Results: A total of 18,201 patients were enrolled and followed for a median of 1.8 years. The mean age was 70 years, $35 \%$ were female and the mean $\mathrm{CHADS}_{2}$ score was 2.1. For the warfarin group, the mean INR TTR was $62 \%$, which is comparable to other trials. ${ }^{7,8}$ The rate of the primary outcome, 
$1.27 \%$ per year in the apixaban group compared with $1.60 \%$ per year in the warfarin group, met the criteria for noninferiority $(p<0.001)$ and superiority $(p=0.01)$. Furthermore, death from any cause was statistically significantly lower in the apixaban group $(3.52 \%$ vs $3.94 \%$ per year; $p=$ $0.047)$. With respect to safety, major bleeding was significantly lower with apixaban compared with warfarin $(2.13 \%$ vs $3.09 \%$ per year; $p<0.001)$, as well as ICH ( $0.33 \%$ vs $0.80 \%$ per year; $p<0.001)$. There was no difference in the rate of GI bleeding between groups.

Implications for practice: In this trial population, apixaban was superior to warfarin in the reduction of stroke and systemic embolism, with an observed reduction in mortality that has not been demonstrated with the other novel oral anticoagulants to date. With less major bleeding, including a lower rate of $\mathrm{ICH}$, apixaban offers some important advantages over warfarin; however, it does require twice-daily dosing. Apixaban was just approved for use in Canada for the indication of prevention of stroke or systemic embolism in patients with AF on December 5, 2012, and thus will likely have a considerable impact on prescribing practices in the future. ${ }^{9}$

\section{Validation of risk stratification schemes for} predicting stroke and thromboembolism in patients with atrial fibrillation: Nationwide cohort study (BMJ 2011)

Background: The $\mathrm{CHADS}_{2}$ score is a common clinical tool for stratifying the annual risk of stroke in patients with nonvalvular AF. The $\mathrm{CHA}_{2} \mathrm{DS}_{2}$ VASc score includes additional risk factors (vascular disease, age 65-74 years, female sex). Neither of these clinical tools has been validated in a large, real-world cohort of AF patients. The purpose of this retrospective analysis of a national patient registry in Denmark ${ }^{4}$ was to investigate predictors of thromboembolism (cardioembolic stroke or systemic embolism) in a cohort of AF patients and to evaluate the predictive value of the $\mathrm{CHADS}_{2}$ and $\mathrm{CHA}_{2} \mathrm{DS}_{2}$-VASc scores.

Patients: Patients with nonvalvular AF or AFL not receiving treatment with a vitamin $\mathrm{K}$ antagonist (VKA) who were discharged from hospital over a 9-year period were included. Patients were excluded if an event (death or thromboembolism) occurred within 7 days of hospital discharge or if patients received a VKA or heparin 6 months prior to hospital admission.
Analysis: Individual components of the $\mathrm{CHADS}_{2}$ and $\mathrm{CHA}_{2} \mathrm{DS}_{2}$-VASc scores were identified using prescription records and diagnosis coding. Outcome rates were estimated based on various combinations of the components for each risk score using Cox regression models at 1, 5 and 10 years of follow-up.

Outcomes: The primary outcome was a composite of death or admission to the hospital due to a thromboembolic event and the secondary outcome was all-cause death.

Results: The cohort consisted of 73,538 patients. Of the patients who were deemed "low risk" by the $\mathrm{CHADS}_{2}$ score (score of 0 ), $40 \%$ were considered "intermediate risk" (score of 1 ) and $22 \%$ were considered "high risk" (score of $\geq 2$ ) by the $\mathrm{CHA}_{2} \mathrm{DS}_{2}$-VASc criteria. Of the patients categorized as being $\mathrm{CHADS}_{2}$ "intermediate risk," 93\% were "high risk" per the $\mathrm{CHA}_{2} \mathrm{DS}_{2}$ VASc score. Patients at "low risk" by the $\mathrm{CHA}_{2} \mathrm{DS}_{2}$-VASc criteria had lower rates of the primary outcome than the corresponding $\mathrm{CHADS}_{2}$ group. The authors concluded that the $\mathrm{CHA}_{2} \mathrm{DS}_{2}$-VASc score was superior to $\mathrm{CHADS}_{2}$ when categorizing patients as low, intermediate or high risk (based on a predictive score known as the C-statistic) and better identified the patients at "truly low risk" of death or a thromboembolic event.

Implications for practice: The $\mathrm{CHADS}_{2}$ score is a common, easy-to-use clinical tool for predicting thromboembolism in patients with nonvalvular AF/AFL. However, the results of this study show it may perform less well at stratifying patients into low-, intermediate- and high-risk groups compared with the $\mathrm{CHA}_{2} \mathrm{DS}_{2}$-VASc score. The $\mathrm{CHA}_{2} \mathrm{DS}_{2}$-VASc score uses additional risk factors to reassess $\mathrm{CHADS}_{2}$ "low-risk" patients to identify those who may benefit from anticoagulant therapy. However, the results are qualified by the inherent limitations of the retrospective observational design. Clinicians managing patients with nonvalvular AF/AFL should be aware of the utility of the $\mathrm{CHA}_{2} \mathrm{DS}_{2}$-VASc score in assessing the risk of thromboembolic events, particularly in patients perceived to be "low risk" according to the $\mathrm{CHADS}_{2}$ score. The 2012 focused update to the Canadian Cardiovascular Society (CCS) AF guidelines now advocates components of the $\mathrm{CHA}_{2} \mathrm{DS}_{2}$-VASc score in their summary of recommendations for choice of antithrombotic agent. $^{10}$ 


\section{ACUTE CORONARY SYNDROMES}

ATLAS ACS 2-TIMI 51: Rivaroxaban in patients with a recent acute coronary syndrome ( $\mathrm{N}$ Engl J Med 2012)

Background: The purpose of this multicentre, randomized, double-blind, placebo-controlled trial was to determine whether the addition of rivaroxaban to standard antiplatelet therapy in acute coronary syndrome (ACS) patients reduces cardiovascular (CV) death, myocardial infarction (MI) or stroke. ${ }^{5}$

Patients: Included were patients with an ACS (ST- or non-ST-segment elevation MI or unstable angina). Patients younger than 55 years also must have had either diabetes mellitus or a previous MI. Patients with a high risk of bleeding, previous $\mathrm{ICH}$ or severe renal dysfunction $(\mathrm{CrCl}<30 \mathrm{~mL} / \mathrm{min})$ were excluded.

Intervention and control: All patients received standard therapy for the treatment of ACS, including acetylsalicylic acid (ASA) and a thienopyridine (e.g., clopidogrel). Patients in the intervention group received rivaroxaban 2.5 $\mathrm{mg}$ or $5 \mathrm{mg}$ twice daily compared with matching placebo in the control group. Of note, the recommended rivaroxaban dose for $\mathrm{AF}$ is $20 \mathrm{mg}$ once daily. $^{2}$

Outcomes: The primary efficacy outcome was a composite of death from CV causes, MI and stroke. Secondary efficacy outcomes included CV and all-cause death. The primary safety end point was major bleeding not related to bypass surgery.

Results: In total, 15,526 patients were enrolled, $75 \%$ of whom were male, with a mean age of 62 years. In comparison to placebo, both the 2.5 $\mathrm{mg}$ and $5 \mathrm{mg}$ doses of rivaroxaban significantly reduced the primary efficacy outcome by approximately $1.8 \%$ ( $8.9 \%$ vs $10.7 \%$; relative reduction of $16 \%$ ) over 13 months. As well, the $2.5 \mathrm{mg}$ dose reduced $\mathrm{CV}$ and all-cause mortality. However, the primary safety end point was significantly higher with both doses of rivaroxaban, although lower with the $2.5 \mathrm{mg}$ dose ( $1.8 \%$ vs $2.4 \%$ with the $5 \mathrm{mg}$ dose), when compared with placebo $(0.6 \%)$. In addition, there was a 3- to 4-fold increase in ICH with both rivaroxaban doses. Minor bleeding was significantly higher with the $5 \mathrm{mg}$ dose but not with the $2.5 \mathrm{mg}$ dose.

Implications for practice: This trial demonstrated that low-dose, twice-daily rivaroxaban, in addition to ASA and a thienopyridine, reduced $\mathrm{CV}$ events and death in patients with ACS. However, this came at the expense of higher rates of major bleeding and $\mathrm{ICH}$, even though the patients in this study were relatively young and those at high risk of bleeding were excluded. The $2.5 \mathrm{mg}$ dose demonstrated better efficacy with less bleeding compared with the $5 \mathrm{mg}$ dose. In practice, it is debatable as to whether this small incremental benefit will outweigh the risk of clinically significant bleeding. Some clinicians may wish to use this therapy in a select group of patients with extensive coronary artery disease and low bleeding risk. In addition to rivaroxaban not currently being indicated for the treatment of ACS in Canada, the $2.5 \mathrm{mg}$ tablet is not yet available-this will likely limit its uptake as an off-label therapy.

\section{HEART FAILURE}

EMPHASIS-HF: Eplerenone in patients with systolic heart failure and mild symptoms (N Engl J Med 2011)

Background: Previous clinical trials have shown that initiating an aldosterone antagonist in patients with systolic HF can reduce mortality and hospitalizations. ${ }^{11,12}$ However, these trials did not investigate the use of aldosterone antagonists in HF patients with mild symptoms (i.e., New York Heart Association [NYHA] class II). The objective of this multicentre, randomized, double-blind, placebo-controlled trial was to determine the benefit and safety of eplerenone in patients with systolic HF and NYHA class II symptoms. ${ }^{6}$

Patients: Enrolled were patients $>55$ years of age with NYHA class II systolic HF (LVEF $\leq 30 \%$ ) who were receiving optimal HF pharmacotherapy with an angiotensinconverting enzyme inhibitor or angiotensin receptor blocker and a beta-blocker at target or maximally tolerated doses. Patients were excluded if they had a serum potassium level $>5$ $\mathrm{mmol} / \mathrm{L}$, severe renal dysfunction (estimated glomerular filtration rate $[\mathrm{eGFR}]<30 \mathrm{~mL} / \mathrm{min}$ ), 
an indication for potassium-sparing diuretics or a recent MI.

Intervention and control: The intervention group was started on eplerenone $25 \mathrm{mg}$ once daily, which was titrated to $50 \mathrm{mg}$ daily after 4 weeks if their serum potassium was $\leq 5.0 \mathrm{mmol} / \mathrm{L}$. Those with an eGFR from $30-50 \mathrm{~mL} / \mathrm{min}$ were initially started on $25 \mathrm{mg}$ every second day, then increased to 25 mg daily after 4 weeks. The control group received matching placebo.

Outcomes: The composite primary end point was the incidence of CV mortality or first hospitalization for HF. Secondary end points included hospitalization for any reason and death from any cause.

Results: In total, 2737 patients were enrolled and followed for a median duration of 1.8 years. The mean age was 69 years and $78 \%$ were male. There was a statistically significant reduction of $7.6 \%$ ( $18.3 \%$ vs $25.9 \%$; relative reduction of $37 \%$ ) in the primary end point favouring eplerenone. The secondary end points of hospitalization for any reason and death from any cause were also significantly reduced with eplerenone. With respect to safety, the incidence of hyperkalemia (serum potassium $>5.5 \mathrm{mmol} / \mathrm{L}$ ) was significantly higher with eplerenone ( $11.8 \%$ vs $7.2 \%, p<0.001)$. The incidence of hypotension, renal failure or gynecomastia was not different between groups.

Implications for practice: This study demonstrated the benefit of eplerenone in systolic HF patients with NYHA class II symptoms and has resulted in this therapy being recommended in the 2011 CCS HF guidelines. ${ }^{13}$ However, this recommendation has a few caveats. One is whether this benefit is a class effect, as the cost of eplerenone is considerably higher than spironolactone. Another important issue is the increased risk of hyperkalemia, as all patients with an elevated serum potassium or taking potassium-sparing diuretics were excluded. Furthermore, although the incidence of renal failure was not different between groups, all patients with severe renal dysfunction were excluded. Therefore, eplerenone should be initiated only in patients at low risk for hyperkalemia and renal dysfunction who can be monitored closely by their health care provider.

From the Mazankowski Alberta Heart Institute, Pharmacy Services, Alberta Health Services, Edmonton, Alberta.Contact arden.barry@albertahealthservices.ca.

\section{References}

1. Statistics-Heart and Stroke Foundation of Canada website. Available: www.heartandstroke.com/site/c.ikIQLcMWJtE/b. 3483991/k.34A8/Statistics.htm\#atrialfib (accessed July 12, 2012).

2. Patel MR, Mahaffey KW, Garg J, et al. Rivaroxaban versus warfarin in nonvalvular atrial fibrillation. $N$ Engl J Med 2011;365:883-91.

3. Granger CB, Alexander JH, McMurray JJV, et al. Apixaban versus warfarin in patients with atrial fibrillation. $N$ Engl $J$ Med 2011;365:981-92.

4. Olesen JB, Lip GY, Hansen ML, et al. Validation of risk stratification schemes for predicting stroke and thromboembolism in patients with atrial fibrillation: nationwide cohort study. BMJ 2011;342:d124.

5. Mega JL, Braunwald E, Wiviott SD, et al. Rivaroxaban in patients with a recent acute coronary syndrome. N Engl J Med 2012;366:9-19.

6. Zannad F, McMurray JJV, Krum H, et al. Eplerenone in patients with systolic heart failure and mild symptoms. $N$ Engl J Med 2011;364:11-21.

7. Connolly SJ, Ezekowitz MD, Yusuf S, et al. Dabigatran versus warfarin in patients with atrial fibrillation. $N$ Engl $J$ Med 2009;361:1139-51.
8. The ACTIVE Writing Group on behalf of the ACTIVE Investigators. Clopidogrel plus aspirin versus oral anticoagulation for atrial fibrillation in the atrial fibrillation clopidogrel trial with irbesartan for prevention of vascular events (ACTIVE W): a randomised controlled trial. Lancet 2006;367:1903-12.

9. Notice of Compliance Information-Health Canada website. Available: http://webprod5.hc-sc.gc.ca/noc-ac/info .do?no=13925\&lang=eng (accessed Jan. 31, 2013).

10. Skanes AC, Healey JS, Cairns JA, et al. Focused 2012 update of the Canadian Cardiovascular Society atrial fibrillation guidelines: recommendations for stroke prevention and rate/ rhythm control. Can J Cardiol 2012;28:125-36.

11. Pitt B, Zannad F, Remme WJ, et al. The effect of spironolactone on morbidity and mortality in patients with severe heart failure. N Engl J Med 1999;341:709-17.

12. Pitt B, Remme W, Zannad F, et al. Eplerenone, a selective aldosterone blocker, in patients with left ventricular dysfunction after myocardial infarction. N Engl J Med 2003;348:1309-21. 13. McKelvie RS, Moe GW, Cheung A, et al. The 2011 Canadian Cardiovascular Society heart failure management guidelines update: focus on sleep apnea, renal dysfunction, mechanical circulatory support, and palliative care. Can J Cardiol 2011;27:319-38. 